\title{
BMJ Open While you're waiting, a waiting room- based, cardiovascular disease-focused educational program: protocol for a randomised controlled trial
}

\author{
Daniel Mcintyre (D) , ${ }^{1}$ Aravinda Thiagalingam, ${ }^{1,2}$ Clara Chow ${ }^{1,2}$
}

To cite: Mcintyre $D$,

Thiagalingam A, Chow C. While you're waiting, a waiting roombased, cardiovascular diseasefocused educational program: protocol for a randomised controlled trial. BMJ Open 2020;10:e036780. doi:10.1136/ bmjopen-2020-036780

- Prepublication history and additional materials for this paper is available online. To view these files, please visit the journal online (http://dx.doi. org/10.1136/bmjopen-2020036780).

Received 07 January 2020 Revised 17 August 2020 Accepted 08 September 2020

Check for updates

(C) Author(s) (or their employer(s)) 2020. Re-use permitted under CC BY-NC. No commercial re-use. See rights and permissions. Published by BMJ.

${ }^{1}$ Westmead Applied Research Centre, University of Sydney,

Westmead, New South Wales,

Australia

${ }^{2}$ Cardiology Department,

Westmead Hospital, Westmead, New South Wales, Australia

Correspondence to

Daniel Mcintyre;

daniel.mcintyre@sydney.edu.au

\section{ABSTRACT}

Introduction Patients with cardiovascular disease (CVD) frequently attend outpatient clinics and spend a significant amount of time in waiting rooms. Currently, this time is poorly used. This study aims to investigate whether providing CVD and cardiopulmonary resuscitation (CPR) education to waiting patients in a cardiology clinic of a large referral hospital improves motivation to change health behaviours, CPR knowledge, behaviours and clinic satisfaction post clinic, and whether there is any impact on reported CVD lifestyle behaviours or relevant CPR outcomes at 30 days.

Methods and analysis Randomised controlled trial with parallel design to be conducted among 330 patients in the waiting room of a chest pain clinic in a tertiary referral hospital. Intervention $(n=220)$ participants will receive a tablet-delivered series of educational videos catered to self-reported topics of interest (physical activity, blood pressure, diet, medications, smoking and general health) and level of health knowledge. Control ( $n=110)$ participants will receive usual care. In a substudy, intervention participants will be randomised 1:1 to receive an extra video on CPR or no extra video. The primary outcome will be the proportion of intervention and control participants who report high motivation to improve physical activity, diet and blood pressure monitoring at end of clinic. The primary outcome of the CPR study will be confidence to perform CPR post clinic. Secondary analysis will examine impact on clinic satisfaction, lifestyle behaviours, CPR knowledge and willingness to perform CPR post clinic and at 30-day follow-up.

Ethics and dissemination Ethics approval has been received from the Western Sydney Local Health District Human Research Ethics Committee. All patients will provide informed consent via a tablet-based eConsent framework. Study results will be disseminated via the usual channels including peer-reviewed publications and presentations at national and international conferences. Trial registration number ANZCTR12618001725257.

\section{INTRODUCTION}

The major risk factors for cardiovascular disease (CVD) are considered the largest contributors to global disease burden, ${ }^{1}$ and coronary heart disease is the single leading cause of death in Australia. ${ }^{2}$ Low survival

\section{Strengths and limitations of this study}

- This study is a randomised controlled trial evaluating the feasibility and impact of a cardiovascular disease-focused educational programme delivered to patients in the waiting room of a rapid access cardiology clinic within a tertiary hospital in Sydney, Australia.

- The intervention is a series of educational videos customised to participant-reported level of health knowledge and topic of interest and will be compared with a control of usual care.

- The primary outcome will be participant-reported motivation to improve cardiovascular health-related lifestyle behaviours immediately post clinic visit.

- A nested study will assess whether a brief educational video on compression-only cardio-pulmonary resuscitation (CPR) will improve confidence and willingness to perform CPR.

- The study will be conducted in a single centre, the intervention delivered only in English and the outcomes will be measured using self-report Likert scales.

rates for out of hospital cardiac arrest is also an important contributor to CVD mortality. ${ }^{34}$

Low educational attainment and poor health literacy have consistently been associated with higher CVD risk, ${ }^{5}$ and the development of educational interventions to support people at risk of CVD is a priority area across many peak CVD health bodies globally. ${ }^{6-8}$ Multiple theoretical models have been proposed to explain the impact (or otherwise) of educational CVD prevention interventions on health attitudes and behaviours. ${ }^{9}$ One example raised in the WHO's 2012 health education guide is the rational or 'knowledge, attitudes and practice' model, in which it is suggested that patient knowledge directly influences health attitudes and practices on a continuum. ${ }^{1011}$ This theoretical basis appears largely consistent with randomised clinical trial data. For 
example, brief educational interventions in cardiopulmonary resuscitation (CPR) improve knowledge, confidence and willingness to perform CPR. ${ }^{12}{ }^{13}$ There is also an extensive literature that describes the potential for brief educational interventions to impact smoking and alcoholrelated attitudes and behaviours. ${ }^{14}{ }^{15}$ However, within most clinical settings, there is insufficient time to deliver important health-related education and promotion. Patients who attend health services spend large amount of time in waiting rooms waiting for their appointment. ${ }^{16}$ This time burden is growing, poorly used and is cumulative for patients with chronic health conditions that frequently attend healthcare appointments. ${ }^{17}$ Yet, this time also presents an opportunity to deliver health education to patients at risk of CVD with little to no time cost for clinicians or patients.

Historically, the clinic waiting room has been used to distribute health education and prevention material with little strategic approach. ${ }^{18}$ The efficacy of some specific approaches to waiting room education, such as printed posters/pamphlets, provision of screening assessments or educational programmes in waiting rooms, have been investigated. ${ }^{19}$ These have suggested some benefits of waiting room-based interventions. For example, Warner $e t$ al (2008) ran a multicentre cluster randomised controlled trial of 38635 patients in three sexual health clinics in the USA and found that a 23-minute video of patientcentred vignettes promoting safe sexual behaviour was effective in reducing sexually transmitted infections (HR $0.91,95 \%$ CI 0.84 to 0.99$).{ }^{20}$ Data from non-randomised studies also suggest provision of education in the waiting room improves patient satisfaction. ${ }^{21-23}$ However, little is known about the impact of these interventions on health attitudes and behaviours. Berkhout et al undertook a systematic review of 21 audio-visual interventions in primary care waiting rooms. They found evidence for knowledge improvement, yet no evidence of impact on health behaviours or attitudes, but there were a number of methodological limitations noted. ${ }^{24}$

There is a paucity of research investigating the impact of education in waiting rooms related to CVD prevention and CPR training. A small study $(\mathrm{n}=100)$ in an emergency department waiting room found participants exposed to a 1-minute video were more likely to perform CPR correctly, indicating such interventions may be feasible and effective for CVD prevention as well. ${ }^{25}$ We hypothesise that patients attending outpatient cardiology clinics, having recently experienced a potential cardiac event, will be highly motivated to engage in both CVD and CPR education. A 2018 Australian qualitative study of cardiac patients and their spouses ( $\mathrm{n}=12$ pairs) found there is strong interest in CPR training among this population, with only one patient-spouse pair stating they had no interest in CPR training. ${ }^{26}$

This study aims to examine in a pragmatic, singleblinded randomised controlled trial of patients in the waiting room of an outpatient cardiology clinic whether waiting room-based CVD prevention educational videos

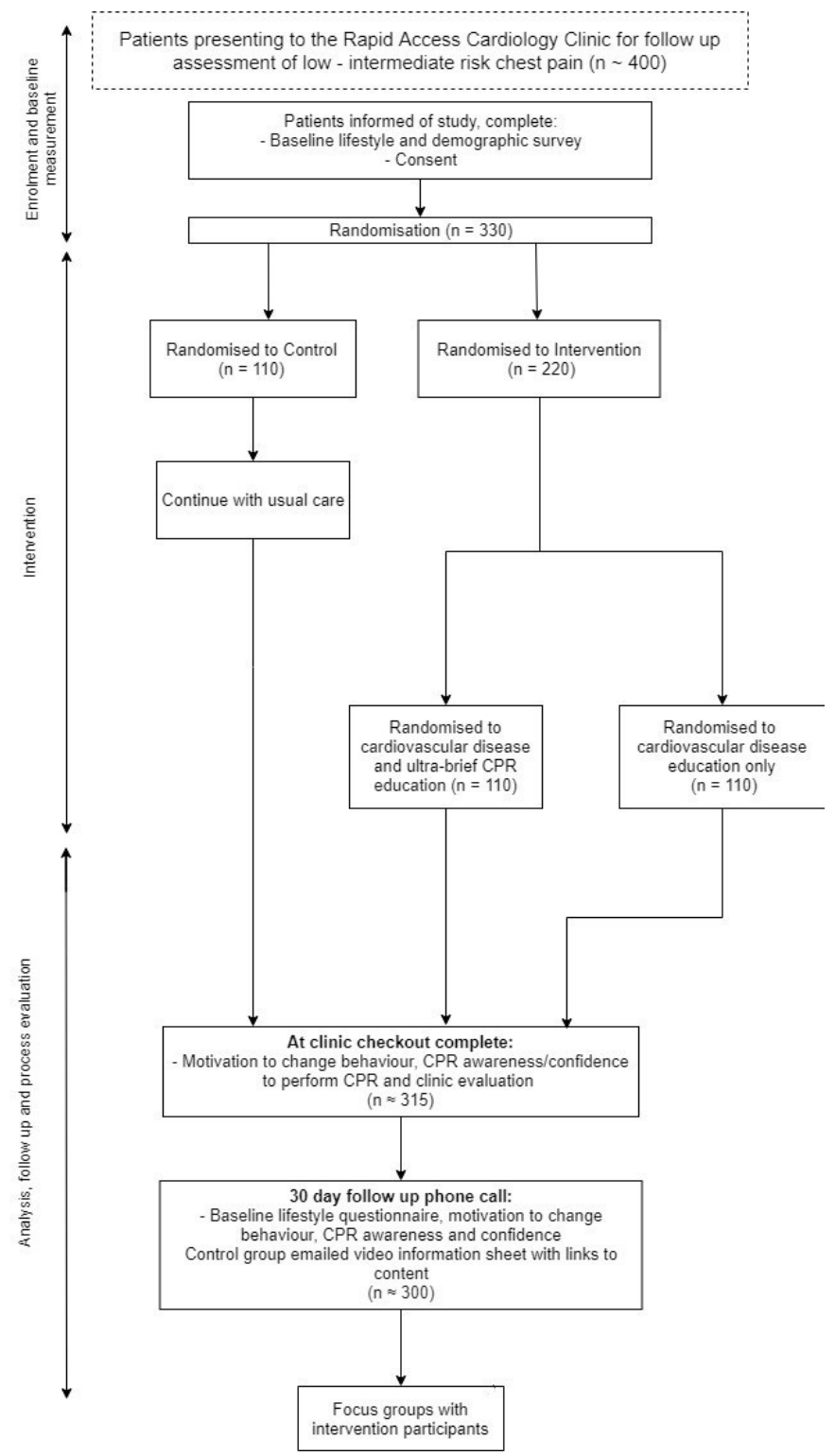

Figure 1 Study Consolidated Standards of Reporting Trials (CONSORT) diagram. CPR, cardiopulmonary resuscitation.

compared with usual care can increase patient motivation to improve cardiovascular health, satisfaction with health services and CVD health-related behaviours. In addition, to specifically examine educational videos impact on CPR knowledge and confidence, intervention participants will be randomly split into two groups with one group to receive an additional CPR video.

\section{METHODS}

\section{Study design}

A single-centre, single-blind, parallel designed randomised controlled trial of 330 patients in the waiting room of an outpatient cardiology clinic within a tertiary teaching hospital in Sydney, Australia (figure 1). Intervention $(\mathrm{n}=220)$ participants will receive a tabletdelivered curated series of advertisement free, web-based 
educational videos covering multiple CVD-related topics. Control $(n=110)$ participants will receive usual care.

Among intervention participants, a second randomisation into two groups will occur, with one group to receive an additional 2-minute educational video on chest only CPR $(n=110)$ or no additional video $(n=110)$. Patients will be assessed post clinic and at 30 days for measures of motivation to improve lifestyle behaviours, clinic satisfaction, actual lifestyle behaviours and knowledge of/confidence to perform CPR.

\section{Patient population}

The study population will be patients presenting to the Rapid Access Cardiology (RAC) Clinic Westmead Hospital. The RAC is a clinic providing specialist assessment of low-intermediate risk chest pain. Most patients seen are first presentation patients as the clinic's remit is to not conduct long-term follow-up of patients to enable the clinic to continue to provide rapid access to new chest pain patients. ${ }^{27}$ Data from a 2015 audit show $13 \%$ of patients had pre-existing coronary artery disease $(\mathrm{CAD})$ and $7.9 \%$ were diagnosed with new CAD. Many patients presenting to this clinic have multiple CAD risk factors $(81.3 \%$ had $\geq 2$ cardiovascular risk factors of preexisting $\mathrm{CAD}$, chronic renal failure, diabetes, hyperlipidaemia, hypertension, overweight/obesity, smoker). ${ }^{28} \mathrm{We}$ therefore predicted that this patient population would be interested in receiving education on CVD risk factors and motivated to make behavioural changes to improve their health following clinic attendance.

\section{Intervention development, patient and public involvement}

The intervention comprised a set of curated educational videos delivered on tablets with patients able to select content to view. The development of the intervention was through a multistage process involving researchers, clinical personnel and consumers to select the delivery platform, the approach to content selection and the approach to content distribution.

The video content was identified through internet searches and recommendations from a range of individuals. A short list of videos was brought together by investigators applying some simple selection criteria: that they were advertisement free, easily accessible on YouTube and addressed topics relevant to CVD prevention including diet, physical activity, hypertension, smoking, medications and CPR education. Investigators prioritised Australian made videos; however, some high-quality resources from the UK and USA were also included.

Curation of the short-listed videos for the purposes of the study was done with the input of a multidisciplinary group of 11 clinical and research staff (cardiology, dietetics, psychology, exercise physiology, physiotherapy, nursing) at Westmead Hospital, and a convenience sample of 21 consumers with CVD recruited via the Cardiomyopathy Association of Australia. Clinicians and consumer input were obtained by a survey tool asking them to rate videos. Clinicians were asked about content accuracy, educational value, perceived utility, appropriate health literacy, potential to cause anxiety and overall whether they would recommend the video to patients to motivate lifestyle change.

Consumers were asked whether videos were engaging, had potential impact on knowledge and potential to motivate a change in lifestyle. Following this input, five videos were excluded due to complexity of content conveyed, inappropriate tone and potential to cause anxiety in the selected patient population.

The final list of videos included 21 educational videos, 7 on diet, 4 on physical activity, 2 on hypertension, 2 on heart attack, 1 on smoking, 1 on alcohol use, 3 on medications and 1 on CPR. Overall, 11 were assigned to a separate general health category. The mean video length was $3 \mathrm{~min}, 14 \mathrm{~s}$ (range $57 \mathrm{~s}-6 \mathrm{~min}, 50 \mathrm{~s}$ ). Top-rated clinician and consumer videos were assigned as 'staff pick' (see figure 2) and appeared at the top of the video list for intervention participants. Other videos were ordered in descending order according to clinician and consumer rating. Participants scan through the list of videos created by their health knowledge and topic of interest selections and decide which videos they would like to watch with minimal input from research staff.

\section{Participant eligibility}

Inclusion criteria are as follows:

1. Adults over 18 years of age.

2. Present at the RAC Westmead for specialist assessment. Exclusion criteria are as follows:

1. Too unwell (physically or mentally) to complete surveys and/or watch videos.

2. Insufficient English language competency to provide informed consent.

3. Previously recruited to the study.

\section{Recruitment and consent}

Eligible patients will be recruited in the waiting room of the RAC clinic in cooperation with clinic staff who will advise if patients meet exclusion criteria (1) or (2). Trained staff will explain the study to potential participants. Recruitment, intervention delivery and outcome collection will be performed around existing clinic flow

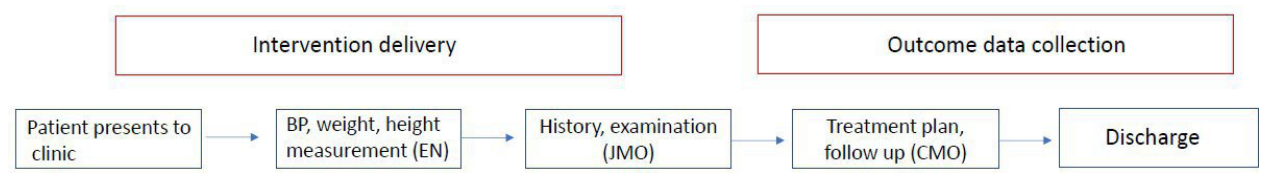

Figure 2 Recruitment procedure: intervention delivery and data collection. CMO, consultant medical officer; EN, enrolled nurse; JMO, junior medical officer. 


\section{WESTMEAD}
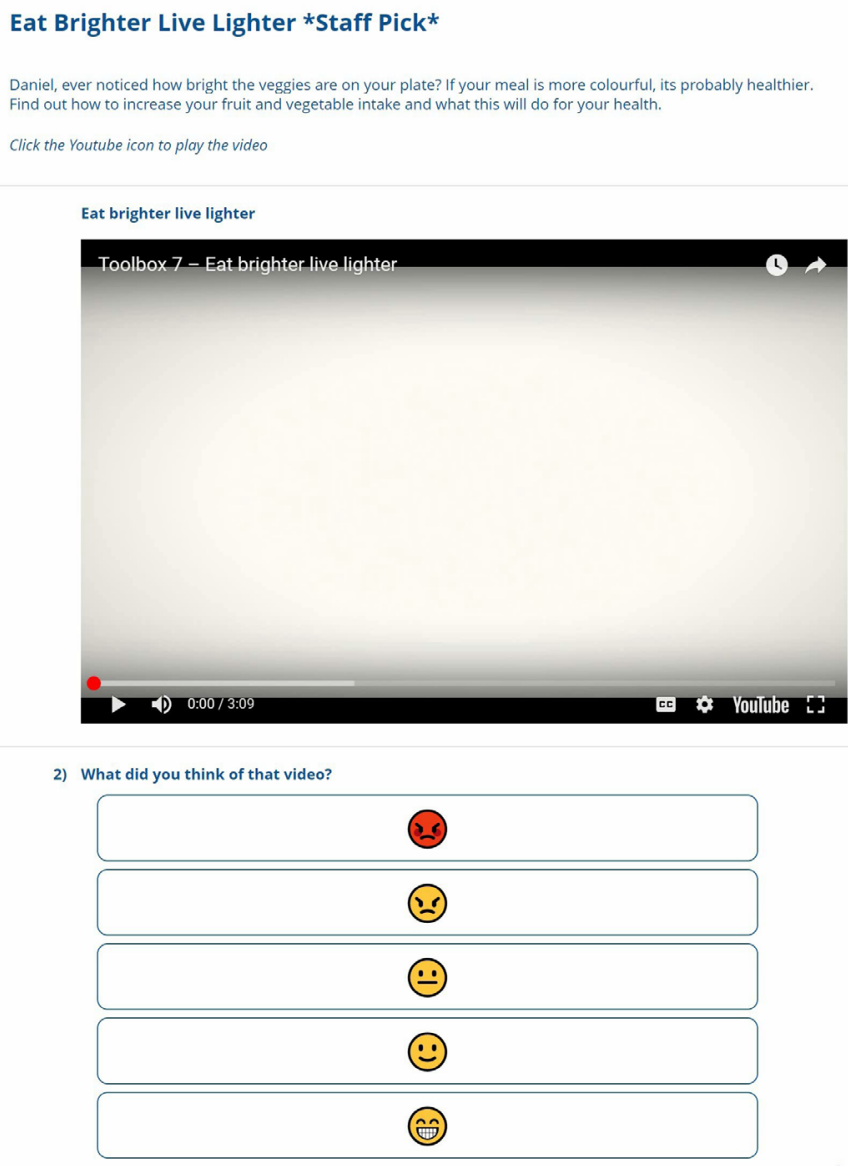

you don't have to respond

3) Any further comments on this video?

Figure 3 Intervention delivery (video reference: https://www. youtube.com/watch?v=v_UPWQze2DU).

such that participation in the study does not increase patient waiting time or affect clinical care (figure 3).

As a waiver of consent was not allowed by the ethics and governance committee overseeing the study, participants will complete eConsent (see online supplemental appendix 1) on iPads provided by the study. The eConsent form will be incorporated into the Research Electronic Data Capture (REDCap) form such that the patient must agree to, complete and sign the eConsent before any data collection or intervention delivery occurs. If patients do not wish to participate, there will be opportunity to decline or withdraw during the clinic day. Ethical approval was obtained from Western Sydney Local Health District Human Research Ethics Committee in December 2018. The first participant was recruited in December 2018.

This trial is registered on the Australian New Zealand Clinical Trails Registry. This includes all items from the WHO Trial Registration Data Set. Final follow-up is anticipated to be complete by April 2020.

\section{Randomisation}

Once participants have consented, the baseline survey (see online supplemental appendix 2) will be initiated on the tablet. Following this, participants will be randomised, with control participants provided a thank you message and intervention participants receiving the initial intervention. Randomisation will occur centrally via a computer-generated sequence, using the randomise $\mathrm{R}$ library of R statistical software (V.3.5.1) and the sequence imported into the REDCap platform. Randomisation will be 2:1 such that there are two intervention patients per one control patient and in randomised permuted blocks of six and three to reduce predictability and ensure a balance between the two arms. Within the intervention arm, participants will be randomised again to receive a CPR video in addition to the other videos or to only receive educational videos.

The randomisation key will be loaded on to the online software platform used to deliver the intervention, the REDCap. ${ }^{29}$ Due to the nature of the intervention, clinic staff and patients cannot be blinded. Study staff recruiting patients and follow-up outcome assessors will be blinded to the condition patients have been randomised to.

\section{Intervention group}

Participants allocated to intervention will be asked about their level of health knowledge (low, medium or high) and topics of interest (physical activity, medications, diet, hypertension, heart attack or general education). This preferences data will be used to generate a customised list of videos using the REDCap queuing feature. Videos are to be delivered through an embedded YouTube link, as if the participants are watching the video online themselves (see figure 3). Prior to receiving the video list, half the participants in the intervention arm will be directed to a 2-minute educational CPR video on YouTube, half will not. Participants will be provided with headphones such that intervention delivery does not disturb others in the waiting room.

\section{Control}

Control participants will only complete the baseline and follow-up surveys and not be offered videos on the tablets and will proceed through usual care in the waiting room. The waiting room in the RAC clinic has some educational posters on the wall, some educational leaflets and a small central television on low volume fixed to a channel chosen by administrative staff. At 30-day follow-up, a copy of the video library with YouTube links will be offered to all control participants.

\section{Study outcomes}

Primary study

The primary outcome is motivation to improve two or more of three CVD prevention behaviours-diet, physical activity and blood pressure monitoring immediately post clinic. Motivation will be assessed with a selfreported 7-point Likert scale. A response of 6 or 7 will be 
considered motivated (see online supplemental appendix $3)$. This outcome will also be assessed as the mean participant score on a continuous scale that combines these three variables (out of a maximum score of 21), and the mean participant score on each separate scale (out of a maximum score of 7 ).

\section{CPR substudy}

For the CPR substudy, the primary outcome will be confidence to perform CPR immediately post clinic. CPR confidence will be measured on a 5-point Likert Scale, with a response of 4 or 5 considered confident (see online supplemental appendix 3). Mean participant confidence scores out of 5 will also be assessed.

\section{Secondary outcomes}

The secondary outcomes measured at end of clinic will be:

- Satisfaction with clinic experience-self-report Likert scales.

- Willingness to perform CPR-yes/no.

- Knowledge of CPR-true/false.

The secondary outcomes measured at 30-day follow-up will be:

- Motivation to improve physical activity and diet—selfreport Likert Scale.

- Mediation adherence-self-report yes/no.

- Diet—self-report Likert Scale.

- Exercise-self-report Likert Scale.

- CPR confidence-self-report Likert Scale.

- Willingness to perform CPR-yes/no.

- CPR knowledge-true/false.

\section{Data collection and management}

Data are collected at baseline, end of the clinic visit and at 30-day follow-up (table 1).

Baseline data will include demographic information, medical history, behaviour and CVD risk factors and be extracted from participant clinical records and supplemented by a brief baseline questionnaire delivered through the REDCap platform on the iPads.

The end of clinic survey is delivered as a paper-based survey given to participants on exiting the clinic and the 30-day follow-up evaluations will be conducted via phone call by study staff (see online supplemental appendices 2-4). Investigators elected to deliver this as a paper-based survey because it ensured participants were not able to enter data into the end of clinic survey prematurely (ie, prior to completion of their clinic appointment), and ensured tablets were available to recruit subsequent participants.

Additional data collected will include total time spent in clinic as a proxy for clinic waiting time. This is collected by clinic administration staff who log patient arrival and departure time in a centralised hospital computer system. Participant feedback on intervention acceptability will be collected in free text and using a visual satisfaction score (see figure 3). Data will be managed in the RedCap system
Table 1 Timing and mode of collection of data used in the study

\begin{tabular}{llll}
\hline Survey & $\begin{array}{l}\text { Baseline } \\
\text { (pre } \\
\text { clinic) }\end{array}$ & $\begin{array}{l}\text { End } \\
\text { of } \\
\text { clinic }\end{array}$ & $\begin{array}{l}\text { 30-day } \\
\text { follow- } \\
\text { up }\end{array}$ \\
\hline $\begin{array}{l}\text { Demographic information and medical } \\
\text { history and behaviour/risk factors* }\end{array}$ & $\mathrm{x}$ & & \\
$\begin{array}{l}\text { Motivation to improve diet and } \\
\text { increase physical activity (see online } \\
\text { supplemental appendices 3 and 4) }\end{array}$ & $\mathrm{x}$ & $\mathrm{x}$ \\
$\begin{array}{l}\text { Motivation to regularly measure blood } \\
\text { pressure (see online supplemental } \\
\text { appendix 3) }\end{array}$ & $\mathrm{x}$ & \\
$\begin{array}{l}\text { Satisfaction with clinic education, } \\
\text { wait time and clinic overall (see online } \\
\text { supplemental appendix 3) }\end{array}$ & & $\mathrm{x}$ & \\
$\begin{array}{l}\text { Self-reported physical activity, fruit } \\
\text { intake, vegetable intake and medication } \\
\text { adherence (see online supplemental } \\
\text { appendices 2 and 4) }\end{array}$ & $\mathrm{x}$ & & $\mathrm{x}$ \\
$\begin{array}{l}\text { Confidence, willingness to perform CPR } \\
\text { and awareness (see online supplemental } \\
\text { appendices 2-4) }\end{array}$ & $\mathrm{x}$ & $\mathrm{x}$ & $\mathrm{x}$ \\
\hline
\end{tabular}

*Information from online supplemental appendix 2 questions and data routinely collected in clinic.

CPR, cardio-pulmonary resuscitation.

during the trial with programmed range checks and sense checks. Physical source data for the end of clinic survey will be stored in a locked office accessible only by card. Primary outcome data will be monitored against source data. At study completion, data will be exported for analysis. Exported data files will be stored in secure password-protected servers at the University of Sydney in compliance with ethical commitments. As no harm is anticipated from this intervention, we do not require a data safety monitoring board.

\section{Sample size}

We estimated a sample size of 330 (2:1 intervention:control ratio), allowing for $\sim 5 \%$ attrition, two-sided tests, and type 1 error of $5 \%$ will have $80 \%$ power to detect a relative increase of $41 \%$ (relative risk (RR) 1.41) in the proportion reporting they are highly motivated in two of three key behaviours. ${ }^{31}$ That is, an absolute increase of $16.4 \%$, from $40 \%$ to $56.4 \%$. We have assumed that approximately $40 \%$ of control participants would report being highly motivated to change behaviour (equal or greater than 6 on a 7-point Likert Scale) based on data reported by Deci et al on patients undergoing chest pain by specialists having high levels of motivation for lifestyle change (mean self-reported autonomous motivation 6.07 on a 7-point positively skewed Likert Scale approximately 1 week post episode $(\mathrm{n}=252, \mathrm{SD}=0.81)){ }^{32}$

In addition, we estimated a sample size of 220 (1:1 intervention:control ratio), allowing for $\sim 5 \%$ attrition, twosided tests and type 1 error of $5 \%$ will have $80 \%$ power to detect a relative increase of $37 \%$ (RR 1.37) in the intervention arm. That is, an absolute increase of $18.5 \%$, from 
$50 \%$ to $68.5 \%$. We have assumed a control rate of $50 \%$ from a survey of CPR education administered to a convenience sample of 100 visitors entering Westmead Hospital on World Heart Day in September 2018 that found 50\% responded 'agree' or 'strongly agree' to the question 'I am confident in my ability to perform CPR'.

\section{Statistical analysis}

Our analysis will be according to a separate statistical analysis plan that will be finalised prior to study completion, data lock and unblinding. Analysis will follow the principals of intention to treat, with participants analysed in the arm they have been allocated. All outcomes will be assessed in an adjusted analysis ( $\log$ binomial for binary outcomes and analysis of covariance for continuous outcomes), which will be considered the primary approach. All adjusted analyses will include age, sex, total waiting time and educational level as covariates. Where outcome measures are available at baseline, the corresponding baseline value will also be included as a covariate. Intervention and control groups will also be compared in an unadjusted analysis, using a $\chi^{2}$ test for binary outcome measures and independent sample t-tests for continuous outcomes. The interaction of treatment effect and age, gender, education, ethnicity and category of presentation (typical/atypical chest pain, arrhythmia or other) will also be explored. However, the study is not powered for analysis of these subgroups. As we are expecting data missingness for the primary outcome to be low $(<5 \%)$, primary analysis will be a complete-case analysis. There are no planned imputation analyses in the event of missing study data.

\section{Process evaluation}

We aim to assess the reasons for impact, or lack thereof, on outcomes from the intervention. We will monitor screening to recruitment rates and note reasons for nonparticipation. This will be explored through participant reactions to videos during intervention delivery, types and number of videos watched during intervention delivery, surveys disseminated to clinic staff and focus groups conducted with participants after the 1-month follow-up.

We will conduct focus group discussions with a targeted sample of individuals to explore the acceptability and perceived utility of the intervention, factors that impact the success of the intervention, barriers and enablers to uptake of the intervention. This will also explore what information on which videos were preferred and why and whether participants found the intervention easy to access (especially patients from culturally and linguistically diverse background, Aboriginal and Torres Strait Islander people and older patients). In addition, we will seek advice and thoughts on how the intervention can be improved, how other facets of the clinic and the waiting process can be improved and advice on how this programme could be integrated into waiting rooms in other healthcare contexts.
Process evaluation surveys by clinic staff will focus on understanding how well the intervention is as it stands fits into the clinic flow and how this can be improved.

Focus groups will be conducted by trained staff. All focus groups will be recorded, transcribed and key themes identified for detailed qualitative analysis.

\section{DISCUSSION}

Clinic waiting time is an unavoidable part of the healthcare experience. Using this perceived wasted time for provision of education may enhance the clinic experience, enhance the clinical appointment experience by both patient and clinician and have other beneficial outcomes on patient motivation in health behaviour modification and self-management. The proposed study evaluates a waiting room-based cardiovascular education focused programme applied using content curated by clinicians, researchers and consumers of entirely off the shelf and freely available video content in a pragmatic, single-blind randomised controlled trial. Our primary objective is to assess the impact on patient motivation to improve health-related behaviours. However, important learnings will also be obtained on potential secondary benefits on patients and clinician experience and how such interventions can be applied in the waiting rooms.

This study presents a novel use of the traditionally neglected and perceived wasted patient waiting time. This study may have implications on the potential use of a wide variety of types of waiting times that occur across healthcare. Patient waiting times for elective surgery and the emergency department are substantial and increasing across multiple Organisation of Economic Co-operation and Development countries. ${ }^{33-35}$ Observational data from multiple studies in the USA, Europe and Asia also indicate that waiting room time in the primary care and outpatients setting is significant and often exceeds time spent in consultation with physicians. ${ }^{16}{ }^{36-38}$ Some small studies have already suggested that interventions delivered during patient waiting time are beneficial in populations at risk of CVD ${ }^{39-41}$ however, we are not aware of any other studies where educational interventions are specifically delivered to patients with high CVD risk in cardiology clinic waiting rooms.

Using this time to improve patient experience and health is likely to be of high interest to clinic operators in both the private and public sectors. If successful, this concept is scalable, and the specific intervention and approach to development is also potentially significantly scalable. The approach to provision of curated information to patients at a time that they are interested in their health is of potential high yield. In the context of our information bombarded current society context in which information curation by the individual is challenging, it also is likely to be highly socially acceptable.

\section{Potential limitations}

This is a single-site study in one hospital in Western Sydney, Australia. We have taken a pragmatic and 
minimalist approach to data collection to improve uptake and minimise impact in already crowded clinical waiting rooms. Hence, to reduce survey fatigue and maximise the time for intervention delivery in the waiting room, we have not delivered more involved, validated tools for the assessment of motivation, satisfaction with services and healthcare behaviours at baseline, post clinic and in follow-up. These factors could reduce our ability to measure the impact of the intervention. The short follow-up period (30 days) also limits our ability to determine if an impact on outcomes at follow-up translates to a lasting change in behaviour that improves health in the long term. Additionally, there is a potential that our integrated, tablet-based consenting, data collection and intervention delivery tool could deter older patients from using the intervention. We will be assessing shortcomings and potential areas for improvement of the delivery tool in our process evaluation.

\section{ETHICS AND DISSEMINATION}

Ethics approval for this study has been granted by the Western Sydney Local Health District Human Research Ethics Committee. During the consenting and follow-up process, patients will be informed of their right to refuse participation in or withdraw from the study. This will not affect their care. Protocol deviations will be reported to this ethics committee and informed consent will be obtained from all study participants.

Study data will only be accessible by study investigators (DM, AT, CC) and the study statistician. Each study participant will be assigned a unique study identification number such that their name and contact number (required for follow-up) are stored separately to their study data. Authorship will be considered according to the International Committee of Journal Editors guidelines. Study results will be disseminated via the usual forums including peer-reviewed publication and at national and international conferences. Investigators will make full protocols and de-identified study data available on reasonable request and subject to ethical approval.

Acknowledgements The authors would like to thank Simone Marschner for her input and advice in developing the outcomes and statistical analysis approach for the current study.

Contributors DM: Conceptualisation, methodology, software, writingoriginal draft, and funding acquisition. AT: Conceptualisation, methodology, software, writing — review and editing, funding acquisition and supervision. CC: Conceptualisation, methodology, writing — review and editing, funding acquisition, supervision and accepts responsibility as the paper guarantor.

Funding This work is supported by the Agency for Clinical Innovation. CC is supported by a National Health and Medical Research Council of Australia Career Development Fellowship co-sponsored by the National Heart Foundation of Australia.

Competing interests None declared.

Patient consent for publication Not required.

Provenance and peer review Not commissioned; externally peer reviewed.

Supplemental material This content has been supplied by the author(s). It has not been vetted by BMJ Publishing Group Limited (BMJ) and may not have been peer-reviewed. Any opinions or recommendations discussed are solely those of the author(s) and are not endorsed by BMJ. BMJ disclaims all liability and responsibility arising from any reliance placed on the content. Where the content includes any translated material, BMJ does not warrant the accuracy and reliability of the translations (including but not limited to local regulations, clinical guidelines, terminology, drug names and drug dosages), and is not responsible for any error and/or omissions arising from translation and adaptation or otherwise.

Open access This is an open access article distributed in accordance with the Creative Commons Attribution Non Commercial (CC BY-NC 4.0) license, which permits others to distribute, remix, adapt, build upon this work non-commercially, and license their derivative works on different terms, provided the original work is properly cited, appropriate credit is given, any changes made indicated, and the use is non-commercial. See: http://creativecommons.org/licenses/by-nc/4.0/.

ORCID iD

Daniel Mcintyre http://orcid.org/0000-0003-4854-4050

\section{REFERENCES}

1 Wang H, Naghavi M, Allen C, et al. Global, regional, and national life expectancy, all-cause mortality, and cause-specific mortality for 249 causes of death, 1980-2015: a systematic analysis for the global burden of disease study 2015. Lancet 2016;388:1459-544.

2 Moon L, Garcia J, Laws P, et al. Measuring health loss in Australia: the Australian burden of disease study. J Korean Med Sci 2019;34:e61-e.

3 Beck B, Bray J, Cameron P, et al. Regional variation in the characteristics, incidence and outcomes of out-of-hospital cardiac arrest in Australia and New Zealand: results from the Aus-ROC Epistry. Resuscitation 2018;126:49-57.

4 Cartledge S, Saxton D, Finn J, et al. Australia's awareness of cardiac arrest and rates of CPR training: results from the Heart Foundation's HeartWatch survey. BMJ Open 2020;10:e033722.

5 Schultz WM, Kelli HM, Lisko JC, et al. Socioeconomic status and cardiovascular outcomes. Circulation 2018;137:2166-78.

6 Atherton JJ, Sindone A, De Pasquale CG, et al. National heart Foundation of Australia and cardiac Society of Australia and New Zealand: guidelines for the prevention, detection, and management of heart failure in Australia 2018. Heart, Lung and Circulation 2018;27:1123-208.

7 Ponikowski P, Voors AA, Anker SD, et al. 2016 ESC Guidelines for the diagnosis and treatment of acute and chronic heart failure: The Task Force for the diagnosis and treatment of acute and chronic heart failure of the European Society of Cardiology (ESC)Developed with the special contribution of the Heart Failure Association (HFA) of the ESC. Eur Heart J 2016;37:2129-200.

8 Arnett DK, Blumenthal RS, Albert MA, et al. 2019 ACC/AHA guideline on the primary prevention of cardiovascular disease: a report of the American college of cardiology/American Heart association task force on clinical practice guidelines. Circulation 2019;140:e596-646.

9 Winter SJ, Sheats JL, King AC. The use of behavior change techniques and theory in technologies for cardiovascular disease prevention and treatment in adults: a comprehensive review. Prog Cardiovasc Dis 2016;58:605-12.

10 Hou S-I. Health education: theoretical concepts, effective strategies and core competencies. Health promotion practice 2014;15:619-21.

11 Vaidya A, Aryal UR, Krettek A. Cardiovascular health knowledge, attitude and practice/behaviour in an urbanising community of Nepal: a population-based cross-sectional study from Jhaukhel-Duwakot health demographic surveillance site. BMJ Open 2013;3:e002976.

12 Beskind DL, Stolz U, Thiede R, et al. Viewing an ultra-brief chest compression only video improves some measures of bystander CPR performance and responsiveness at a mass gathering event. Resuscitation 2017;118:96-100.

13 Panchal AR, Meziab O, Stolz U, et al. The impact of ultra-brief chest compression-only CPR video training on responsiveness, compression rate, and hands-off time interval among bystanders in a Shopping mall. Resuscitation 2014;85:1287-90.

14 Wray JM, Funderburk JS, Acker JD, et al. A meta-analysis of brief tobacco interventions for use in integrated primary care. Nicotine Tob Res 2018;20:1418-26.

15 Kaner EFS, Beyer FR, Muirhead C, et al. Effectiveness of brief alcohol interventions in primary care populations. Cochrane Database of Systematic Reviews 2018;24.

16 Arain M, Nicholl J, Campbell M. Patients' experience and satisfaction with GP led walk-in centres in the UK; a cross sectional study. BMC Health Serv Res 2013;13:142. 
17 Mclntyre D, Chow CK. Waiting time as an indicator for health services under strain: a narrative review. INQUIRY: The Journal of Health Care Organization, Provision, and Financing 2020;57:0046958020910305.

18 Gignon M, Idris H, Manaouil C, et al. The waiting room: vector for health education? the general practitioner's point of view. BMC Res Notes 2012;5:511.

19 Cass SJ, Ball LE, Leveritt MD. Passive interventions in primary healthcare waiting rooms are effective in promoting healthy lifestyle behaviours: an integrative review. Aust J Prim Health 2016;22:198-210.

20 Warner L, Klausner JD, Rietmeijer CA, et al. Effect of a brief video intervention on incident infection among patients attending sexually transmitted disease clinics. PLoS Med 2008;5:e135.

21 Stribling JC, Richardson JE. Placing wireless tablets in clinical settings for patient education. Journal of the Medical Library Association : JMLA 2016;104:159-64.

22 Papa L, Seaberg DC, Rees E, et al. Does a waiting room video about what to expect during an emergency department visit improve patient satisfaction? CJEM 2008;10:347-54.

23 Reid S, Neto G, Tse S, et al. Education in the waiting room: description of a pediatric emergency department educational initiative. Pediatric emergency care 2017;33:e87-91.

24 Berkhout C, Zgorska-Meynard-Moussa S, Willefert-Bouche A, et al. Audiovisual AIDS in primary healthcare settings' waiting rooms. A systematic review. Eur J Gen Pract 2018;24:202-10.

25 Benoit JL, Vogele J, Hart KW, et al. Passive ultra-brief video training improves performance of compression-only cardiopulmonary resuscitation. Resuscitation 2017:115:116-9.

26 Cartledge S, Feldman S, Bray JE, et al. Understanding patients and spouses experiences of patient education following a cardiac event and eliciting attitudes and preferences towards incorporating cardiopulmonary resuscitation training: a qualitative study. $J$ Adv Nurs 2018;74:1157-69.

27 Chow CK, Timmis A. Rapid access clinics for patients with chest pain: will they work in Australia? Med J Australia 2019;210:307-8.

28 Klimis $\mathrm{H}$, Khan ME, Thiagalingam A, et al. Rapid access cardiology (Rac) services within a large tertiary referral Centre-First year in review. Heart, Lung and Circulation 2018;27:1381-7.
29 Harris PA, Taylor R, Minor BL, et al. The REDCap Consortium: building an international community of software platform partners. $J$ Biomed Inform 2019;95:103208.

30 Harris PA, Taylor R, Thielke R, et al. Research electronic data capture (REDCap) - A metadata-driven methodology and workflow process for providing translational research informatics support. J Biomed Inform 2009;42:377-81.

31 Chapman and Hall/CRC. Epidemiology: study design and data analysis. 3rd edn, 2013.

32 Deci EL, Gagné M, Mushlin Al, et al. Motivation for behavior change in patients with chest pain. Health Education 2005;105:304-21.

33 Siciliani L, Moran V, Borowitz M. Measuring and comparing health care waiting times in OECD countries. Health Policy 2014;118:292-303

34 NHS England. Hospital accident and emergency activity, 2018-2019, 2019.

35 Barua B, Jacques D, Collyer A. Waiting your turn: wait times for health care in Canada, 2018 report. Fraser Insititute, 2018.

36 Patwardhan A, Davis J, Murphy P, et al. Comparison of waiting and consultation times in convenient care clinics and physician offices. $J$ Prim Care Community Health 2013;4:124-8.

37 Luque Ramos A, Hoffmann F, Spreckelsen O. Waiting times in primary care depending on insurance scheme in Germany. BMC Health Serv Res 2018;18:191.

38 Ahmad BA, Khairatul K, Farnaza A. An assessment of patient waiting and consultation time in a primary healthcare clinic. Malays Fam Physician 2017;12:14-21.

39 Jackson RA, Stotland NE, Caughey AB, et al. Improving diet and exercise in pregnancy with video doctor counseling: a randomized trial. Patient Educ Couns 2011;83:203-9.

40 Cohen AJ, Richardson CR, Heisler M, et al. Increasing use of a healthy food incentive: a waiting room intervention among lowincome patients. Am J Prev Med 2017;52:154-62.

41 Yvonne Chan Y-F, Nagurka R, Richardson LD, et al. Effectiveness of stroke education in the emergency department waiting room. $J$ Stroke and Cerebrovas Dis 2010;19:209-15. 